\title{
The Crystal Structure of Klebsiella pneumoniae FeoA Reveals a Site For Protein-Protein Interactions
}

\author{
Richard O. Linkous, Alexandrea E. Sestok, and Aaron T. Smith* \\ Department of Chemistry and Biochemistry, University of Maryland, Baltimore County, Baltimore, Maryland, 21250 USA
}

\begin{abstract}
In order to establish infection, pathogenic bacteria must obtain essential nutrients such as iron. Under acidic and/or anaerobic conditions, most bacteria utilize the Feo system in order to acquire ferrous iron $\left(\mathrm{Fe}^{2+}\right)$ from their host environment. The mechanism of this process, including its regulation, remains poorly understood. In this work, we have determined the crystal structure of FeoA from the nosocomial agent Klebsiella pneumoniae $(K p \mathrm{FeoA})$. Our structure reveals an $\mathrm{SH} 3-$ like domain that mediates interactions between neighboring polypeptides via intercalations into a Leu zipper motif. Using docking of a small peptide corresponding to a postulated FeoB partner binding site, we demonstrate the $K p$ FeoA can assume both 'open' and 'closed' conformations, controlled by peptide binding. We propose a model in which a ' $\mathrm{C}$-shaped' clamp along the FeoA surface mediates interactions with its partner protein, FeoB. These findings are the first to demonstrate atomic-level details of FeoA-based protein-protein interactions, which could be exploited for future antibiotic developments.
\end{abstract}

The acquisition of iron is an essential virulence factor for the establishment of infection by a wide array of bacterial pathogens, ${ }^{1-2}$ including one of the major causative agents of nosocomial (hospital-acquired) infections, Klebsiella pneumoniae. ${ }^{3-4}$ The environmental source of iron is typically the host, where it may be found in multiple oxidation and coordination states, necessitating pathogens such as $K$. pneumoniae to adapt to acquire iron in ferric $\left(\mathrm{Fe}^{3+}\right)$, ferrous $\left(\mathrm{Fe}^{2+}\right)$, and even chelated forms. Under oxidizing conditions, siderophore- and heme-based acquisition systems are essential to stabilize, to solubilize, and to transport ferric iron. ${ }^{1}$ However, under acidic, micro-aerobic, and/or anaerobic conditions, such as those found in the gut or within biofilms, iron may be prevalent in the reduced, ferrous form. ${ }^{5-6}$ Because ferrous iron has differences in solubility, lability, and even coordination properties compared to ferric iron, bacteria such as $K$. pneumoniae must employ orthogonal transport systems to acquire and to handle $\mathrm{Fe}^{2+} \cdot 5-6$
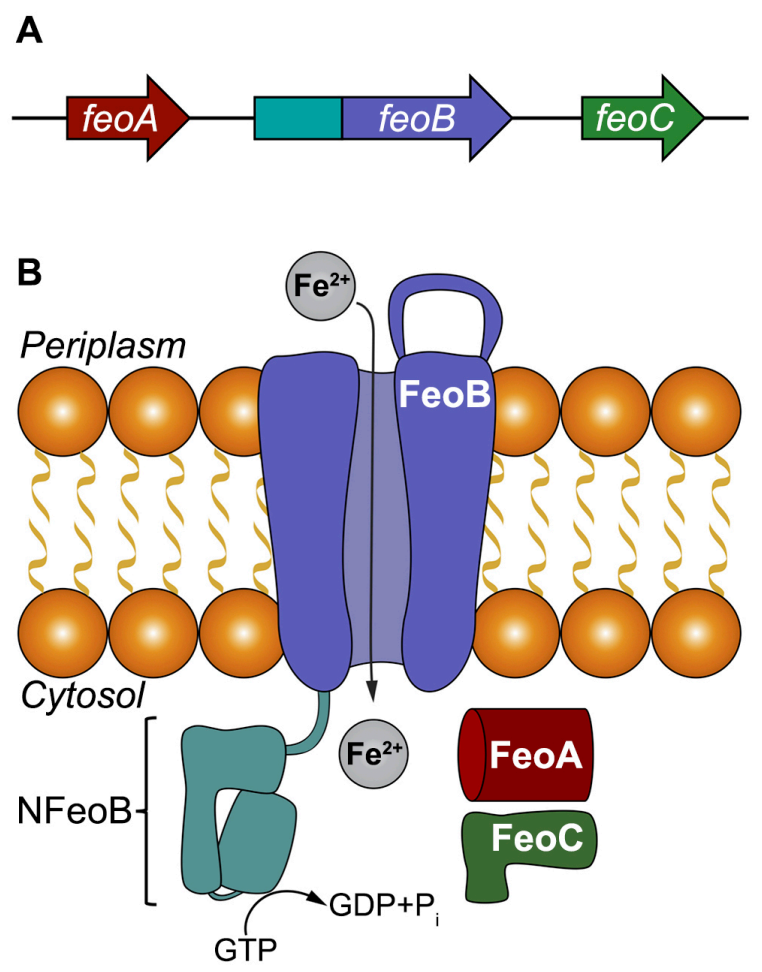

Figure 1. The Feo system in K. pneumoniae. A. The $f e o$ operon within $K$. pneumoniae encodes for three proteins: FeoA, FeoB, and FeoC. B. In K. pneumoniae, FeoA (red) and FeoC (green) are small, cytosolic proteins that are predicted to function as accessories to ferrous $\left(\mathrm{Fe}^{2+}\right)$ transport across the intracellular membrane, mediated by the polytopic membrane protein FeoB (purple). At the N-terminus of FeoB is the soluble GTP-binding domain known as NFeoB (teal), which is capable of hydrolyzing GTP and may drive ferrous iron transport in an active manner.

The most prevalent prokaryotic transport system dedicated to the transport of $\mathrm{Fe}^{2+}$ is the ferrous iron transport system, also known as Feo (Fig. 1). ${ }^{-6}$ In K. pneumoniae, this system is found along the feo operon (Fig. 1A), which encodes for three proteins: FeoA, FeoB, and FeoC. $^{7}$ FeoA and FeoC are predicted to be small $(\sim 8$ $\mathrm{kDa})$, cytosolic proteins, whereas FeoB is a large $(\sim 90$ $\mathrm{kDa}$ ), polytopic membrane protein bearing a $\mathrm{N}$-terminal 
GTP-binding domain (NFeoB; Fig. 1B). These three proteins are postulated to function in concert to regulate the movement of ferrous iron from the periplasm into the cytosol (Fig. 1B), where it is presumably handed off to an unknown ferrous iron chaperone for assembly into iron-containing proteins and/or intracellular storage. ${ }^{6}$

We recently described the preparation and biophysical properties of detergent-solubilized $K$. pneumoniae FeoB $(\mathrm{KpFeoB}) .^{7}$ Of note was the ability of $K p \mathrm{FeoB}$ to hydrolyze GTP at a rate comparable to the hydrolysis of ATP by some $\mathrm{ABC}^{8-9}$ and $\mathrm{P}_{1 \mathrm{~B}}$-ATPase $\mathrm{e}^{10-11}$ metallotransporters, suggesting that ferrous iron transport could be driven in an active manner by GTP hydrolysis. However, this hydrolysis rate $\left(\sim 0.1 \mathrm{~s}^{-1}\right)$ is still considered sluggish by means of most active transporters. ${ }^{7}$ The slow rate of GTP hydrolysis by FeoB has lead us and others to consider that an additional stimulatory factor may exist to upregulate hydrolysis under changing intracellular conditions in order to drive ferrous iron uptake. ${ }^{6-7,12-14}$ It is postulated that this stimulatory factor is FeoA.

Several three-dimensional structures of FeoA exist, ${ }^{13,}$ 15 and these structures reveal the presence of an Srchomology 3 (SH3)-like fold. SH3 folds, which are common to eukaryotes and are characterized by small $(<100$ amino acids) $\beta$-barrels, are often involved in mediating protein-protein interactions and are even utilized to activate eukaryotic GTPases. ${ }^{16-19} \mathrm{SH} 3$ folds typically interact with binding sites on partner proteins bearing a consensus motif of PxxP, with " $\mathrm{x}$ " frequently being a hydrophobic amino acid. ${ }^{20}$ To our knowledge, every FeoB sequence that we have examined contains a candidate PxxP binding site, leading to the consideration that this site is the location for FeoA-FeoB interactions. However, how FeoA may facilitate this interaction remained unknown until now.

In order to investigate the function of $K p \mathrm{FeoA}$, we cloned, expressed, and purified $K p$ FeoA to good yield and high purity (Supplemental Methods; Fig. S1). Circular dichroism studies indicated our overexpressed, purified protein was folded (data not shown), and gel filtration studies demonstrated the presence of two oligomeric species whose molecular weights were consistent with monomeric $(\sim 10 \mathrm{kDa})$ and dimeric $(\sim 20 \mathrm{kDa})$ (His) $)_{6^{-}}$ tagged $K p$ FeoA (Fig. S2). We observed no trimeric or higher-order oligomerization of $K p \mathrm{FeoA}$ under these conditions. Moreover, our oligomeric states appeared to be static under our gel filtration conditions, as dilution of dimeric $K p \mathrm{FeoA}$ and reinjection preserved oligomeric homogeneity (Fig. S2). To characterize these states further, we attempted to crystalize both oligomeric species independently; however, we were only successful in generating diffraction-quality crystals with monomeric $K p \mathrm{FeoA}$ in the crystallization drop.

Crystals of unmodified, tagged $K p$ FeoA diffracted to $<2 \AA$, and our best native dataset was processed to a res- olution of $1.57 \AA$ (Table S1). Despite exhaustive efforts to utilize molecular replacement (MR) to determine phases, including the use of an unpublished NMR structure of $K p \mathrm{FeoA}$, we were unable to find a suitable MR solution, suggesting our structure adopted a conformation distinct from previously determined structures. Subsequently, we expressed, purified, and crystallized SeMet-derived $K p$ FeoA (Supplemental Methods), and were able to establish phases utilizing single-wavelength anomalous dispersion (SAD). This model then was used to establish phases definitively for our native data via MR. Our final refined model converged to an $R_{\text {work }} / R_{\text {free }}$ of $0.174 / 0.193$ (Table S1), and all residues comprising the full length of the $K p$ FeoA polypeptide (1-75) and part of the tag cleavage site (76-80) were unambiguously present in the electron density (Fig. S3).

Initial inspection of a single monomer comprising the
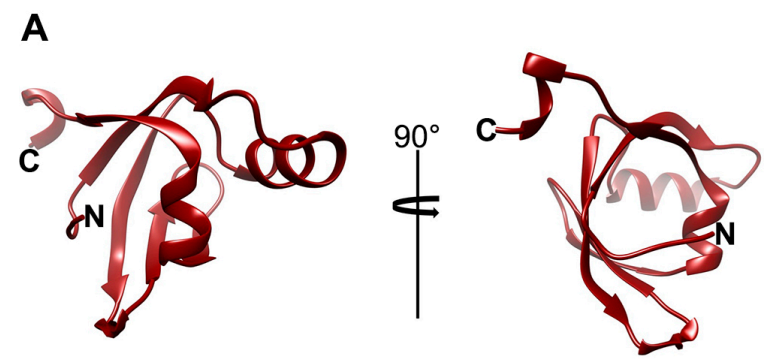

B

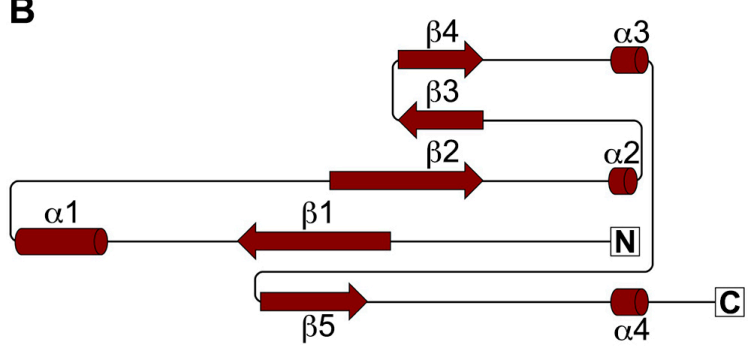

Figure 2. The crystal structure of a single $K p$ FeoA polypeptide (PDB ID 6E55). A. The ribbon structure of $K p \mathrm{FeoA}$ reveals a small $\beta$ barrel comprising an $\mathrm{SH} 3-$ like fold. The right panel represents a $90^{\circ}$ rotation of the left panel. B. Secondary-structure topology diagram of monomeric $K p$ FeoA. $\alpha$ helices and $\beta$ sheets are numbered sequentially. " $\mathrm{N}$ " and " $\mathrm{C}$ " represent the location of the $\mathrm{N}$ - and $\mathrm{C}$-termini, respectively.

$\mathrm{X}$-ray crystal structure of $K p \mathrm{FeoA}$ reveals the expected SH3-like fold present in other FeoA structures (Fig. $2 \mathrm{~A}){ }^{13,}{ }^{15}$ In particular, we observe five $\beta$ strands $(\beta 1-\beta 5)$ that comprise the $\beta$ barrel, complemented by two additional $\alpha$ helices $(\alpha 1, \alpha 3)$ and a helical turn $(\alpha 2)$ that appear to be unique to FeoAs (Fig. 2B). ${ }^{13,15}$ A fourth, short helix $(\alpha 4)$ appears at the C-terminal tail in the visible electron density, but this helix is composed of residues that are part of the tag cleavage site and are not part of the native sequence. 
A

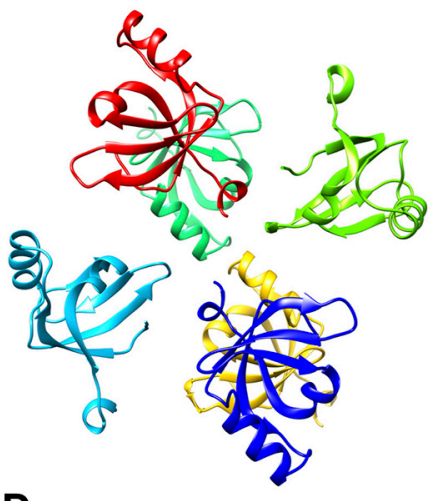

B

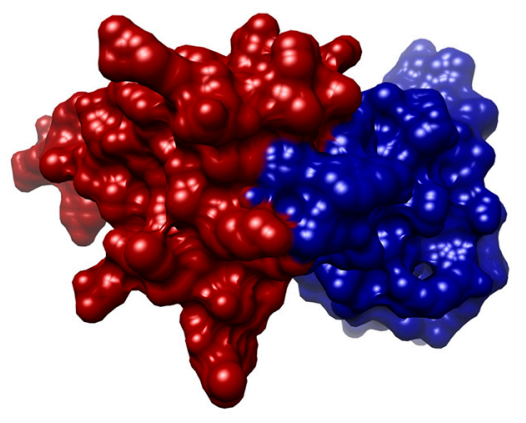

C

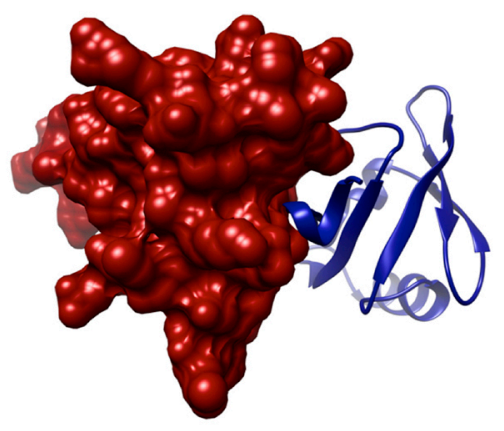

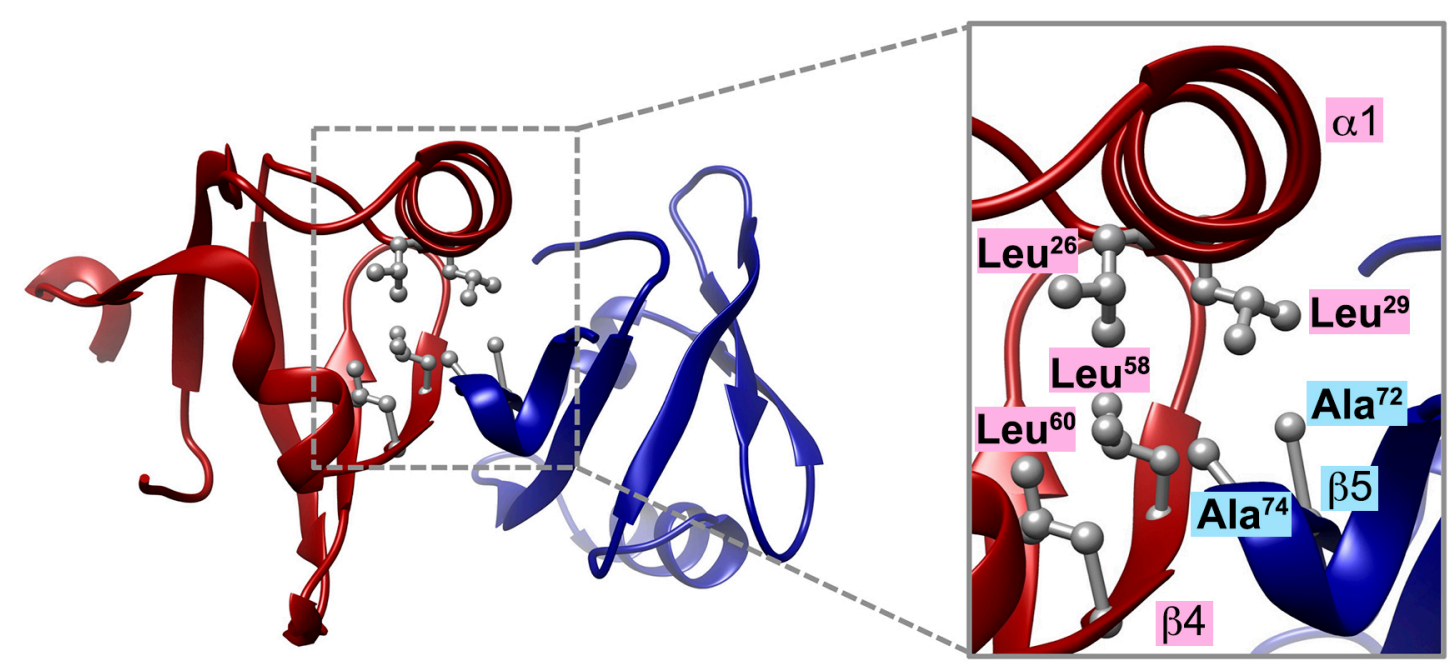

Figure 3. Features of $K p \mathrm{FeoA}$ interactions. A. Ribbon representation of the asymmetric unit (ASU) of the crystal structure of $K p$ FeoA, which contains 6 molecules. B. Space-filling model of two adjacent, interacting $K p$ FeoA polypeptides. The individual polypeptides are colored in red and blue. C. The same two interacting $K p$ FeoA polypeptides in B with one polypeptide displayed as space-filling and the other displayed as ribbon, emphasizing the intercalation of one molecule into the other. D. Ribbon representation of the $K p \mathrm{FeoA}-K p \mathrm{FeoA}$ interaction, with key hydrophobic residues represented as gray balls and sticks. Inset: two Ala residues along $\beta 5\left(\mathrm{Ala}^{72}\right.$ and $\left.\mathrm{Ala}^{74}\right)$ intercalate into a Leu zipper motif comprising 4 Leu residues: Leu ${ }^{26}$ and $\mathrm{Leu}^{29}$ (along $\alpha 1$ ) and $\mathrm{Leu}^{58}$ and $\mathrm{Leu}^{60}$ (along $\beta 4$ ). Leu ${ }^{29}$ becomes displaced from the zipper as it interacts with the Ala residues along $\beta 4$.

Intriguingly, analysis of the $K p$ FeoA asymmetric unit (ASU) composed of 6 polypeptides reveals relevant interactions among neighboring $K p \mathrm{FeoA}$ molecules (Fig. $3 \mathrm{~A}$ ). Within the ASU there are $2 \mathrm{KpFeoA}$ dimers and 2 $K p$ FeoA monomers. Comprising the dimers, two independent $K p$ FeoA chains participate in hydrophobic intercalations into a Leu zipper motif present on their dimeric partner $K p F e o A$ chains, allowing for the exclusion of a modest amount $\left(\sim 29 \AA^{2}\right)$ of hydrophobic surface area (Fig. 3B,C). The Leu zipper motif on a single $K p$ FeoA polypeptide is composed of four residues along a surface ridge that forms a "C-shaped" clamp (Fig. 3D): $\mathrm{Leu}^{26}$ and Leu ${ }^{29}$ (present along $\alpha 1$ ) and Leu ${ }^{58}$ and Leu ${ }^{60}$ (present along $\beta 4$ ). Intercalated into this hydrophobic zipper are two residues of the neighboring $K p F e o A$ monomer along 35 : $\mathrm{Ala}^{72}$ and $\mathrm{Ala}^{74}$ (Fig. 3D). This intercalation appears to displace $\mathrm{Leu}^{29}$ from the central portion of the zipper towards the Ala residues along $\beta 5$ (Fig 3D). This interaction is repeated throughout the crystal (Fig. S4), and thus the two $K p F$ FeoA "monomers" without partners in the ASU interact with the $\beta 5$ of the adjacent ASU, repeating these interactions throughout the entire crystalline lattice.

This interaction results in a significant "closing" or "clamping" of the $K p \mathrm{FeoA} \mathrm{SH3-like} \mathrm{fold} \mathrm{onto} \mathrm{its} \mathrm{neigh-}$ boring polypeptide. We compared our crystal structure to the unpublished NMR structure of $K p$ FeoA (PDB ID $2 \mathrm{GCX}$ ). Superposition of our structure onto $2 \mathrm{GCX}$ chain A shows conservation of the global fold, but there are 
A
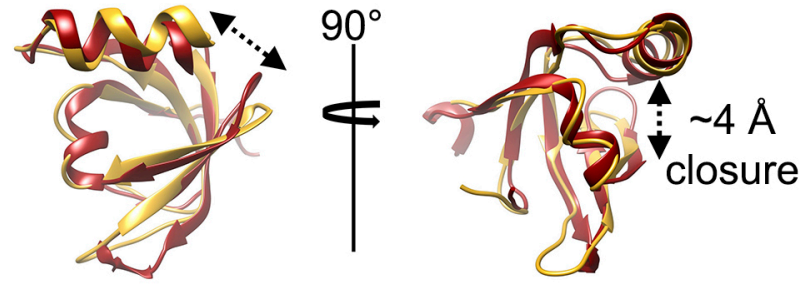

C

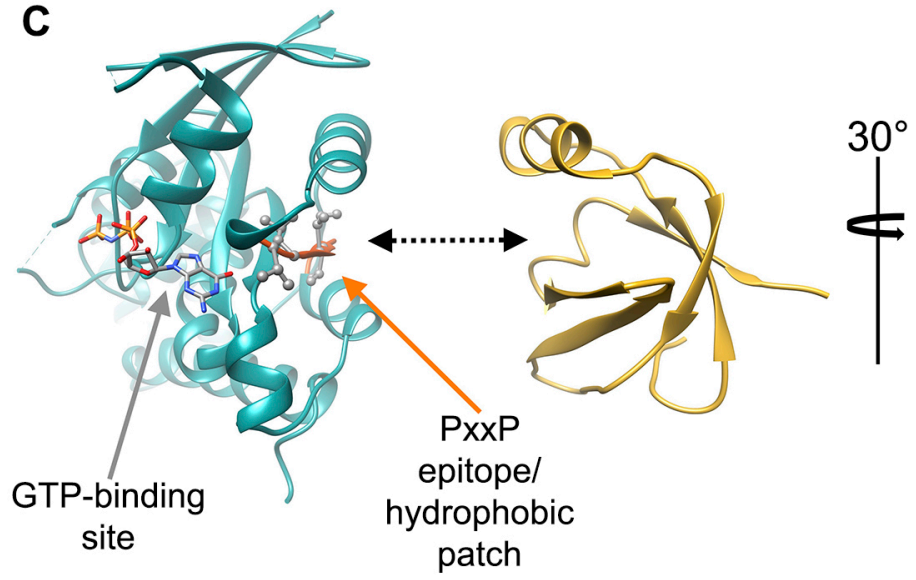

B

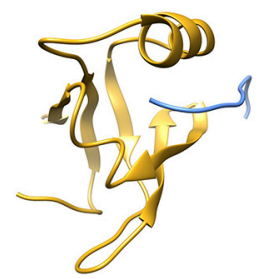

"open"

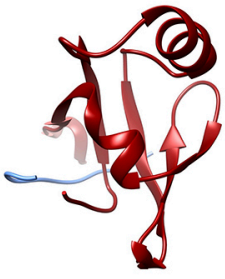

"closed"

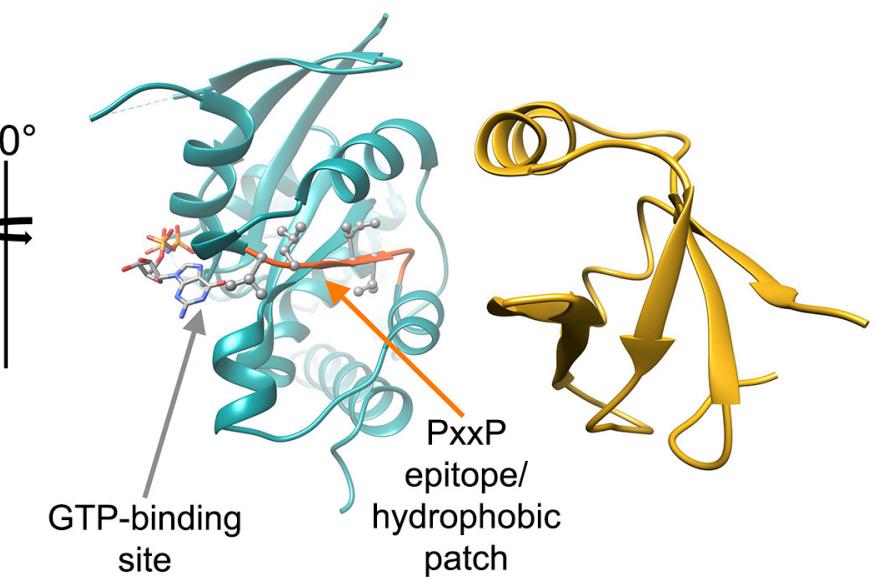

Figure 4. Structural analysis of $K p$ FeoA. A. Superposition of our crystal structure of $K p$ FeoA (red) with the NMR structure of $K p$ FeoA (goldenrod; PDB ID 2GCX). While the global fold is conserved, there is a significant closure $(\sim 4 \AA)$ of the C-terminal side of $\alpha 1$ and the $\beta 3-\beta 4$ turn in our structure compared to the NMR structure. B. Docking studies of the 10 amino acid sequence (cornflower) postulated to be the FeoA recognition site along NFeoB. In the "open" NMR structure (goldenrod), the 10mer peptide docks precisely in the same location we observe interactions between $K p F e o A$ dimers in our crystal structure. In the "closed" crystal structure (red), the 10-mer peptide fails to dock in the "C-shaped" clamp due to the closing of this binding region. C. We hypothesize that the open form of $K p F e o A$ (goldenrod) binds to the PxxP recognition site on NFeoB (orange; Leu and Val residues shown as ball and stick of $K p$ NFeoB bound to GMP-PNP, PDB ID: 2WIC). We postulate this binding can be directly communicated to the GTP-binding site through the short helical loop containing hydrogen bonds to the guanine nucleobase. The right panel of $\mathbf{C}$ represents a $30^{\circ}$ rotation of the left panel of $\mathbf{C}$.

several structural changes resulting in a rmsd of $\sim 2.3 \AA$ over $74 \mathrm{C} \alpha$ atoms (Fig. 4A). This structural deviation likely explains the failure of the NMR model for MR. The most striking structural differences observed are the closing of the space between the $\beta 3-\beta 4$ turn and that of the C-terminal side of $\alpha 1$ (Fig. 4A). The two residues anchoring the ends of this region are $\mathrm{Leu}^{29}$ and $\mathrm{Arg}^{55}$. In the NMR structure, this area is quite open: the distance of $\mathrm{C} \alpha \mathrm{Leu}^{29}$ to $\mathrm{C} \alpha \mathrm{Arg}^{55}$ is $\sim 10.5 \AA$. In stark contrast, our crystal structure reveals that this region has closed along $\beta 5$ of the neighboring molecule: the distance of $\mathrm{C} \alpha \mathrm{Leu}^{29}$ to $\mathrm{C} \alpha \mathrm{Arg}^{55}$ has decreased to $\sim 6.6 \AA$, representing a nearly $4 \AA$ narrowing. Previous analyses of the NMR structure of $E c F e o A$ have indicated dynamicism to be present with this same region and, in particular, along $\beta 4 .{ }^{13}$ Thus, we propose that $2 \mathrm{GCX}$ represents the "open" form of $K p \mathrm{FeoA}$, and that our structure represents the "closed" form of $K p F e o A$ bound to a protein partner.
We believe our structure points to the location along FeoA that may mediate interactions with its corresponding binding site along NFeoB. To test our "open-toclosed" hypothesis, we performed in silico docking experiments $^{21-22}$ of both $K p$ FeoA models with a hydrophobic 10 AA sequence (LGCPVIPLVS) representing the postulated partner binding site present on NFeoB. We excised the structure of this 10-mer directly from the crystal structure of $K p$ NFeoB bound to GMP-PNP (PDB ID 2 WIC). ${ }^{23}$ Wholly consistent with our hypothesis, the lowest-energy docking model of this peptide with the NMR structure of $K p$ FeoA predicts binding directly within the "C-shaped" clamp of the "open" conformer (Fig. 4B). In contrast and as predicted, the lowest-energy docking model of this peptide with our crystal structure of $K p \mathrm{FeoA}$ fails to dock into the now "closed" binding site (Fig. 4B).

In light of these data, we propose a mechanism of FeoA-NFeoB interactions that may link to the status, or alter the state, of bound nucleotide (Fig. 4C). We posit 
bioRxiv preprint doi: https://doi.org/10.1101/514059; this version posted January 8,2019 . The copyright holder for this preprint (which was not certified by peer review) is the author/funder. All rights reserved. No reuse allowed without permission.

that the "open" conformer of $K p \mathrm{FeoA}$ uses its "Cshaped" clamp region (defined by $\alpha 1$ and $\beta 4$ ) to interact with the PxxP recognition site on NFeoB. This location is rich in hydrophobic residues (Val and Leu) that would likely intercalate into the Leu zipper along $K p$ FeoA (Fig. 4C), similar to what we observe in our crystal structure (Fig. 3D). Moreover, hydrophobic residues are strongly conserved at, or adjacent to, the Leu zipper location despite low overall conservation of FeoA sequence, emphasizing the functional importance of hydrophobicity in this vicinity. We envision a scenario in which the FeoANFeoB binding event is communicated to, or even linked to the state of, GTP/GDP bound on the surface of NFeoB. In the both the GMP-PNP- and GDP-bound $K p$ NFeoB structures, the guanine nucleobase is connected via hydrogen bonding directly to the PxxP binding site by a short helical turn. ${ }^{23}$ Thus, FeoA binding at this site could either increase the rate of GTP hydrolysis, facilitate nucleotide release, or both.

Our discovery of a site for FeoA-mediated proteinprotein interactions opens up several exciting avenues for future research on the Feo system. Numerous studies have demonstrated that FeoA is a virtually indispensable component of the bacterial Feo system, ${ }^{24-27}$ likely due to FeoA's regulatory role in modulating ferrous iron import through its interaction with FeoB. Furthermore, the disruption of protein-protein interactions mediated by complex surface recognition sites along SH3-like domains like FeoA is an active area of pharmacological development in eukaryotes. ${ }^{20,28}$ This targeted approach could extend to antibiotic development aimed a nutrient uptake pathways such as Feo. We imagine a future scenario in which small, hydrophobic molecules could be developed to disrupt FeoA-FeoB interactions as a novel means of attenuating bacterial virulence through the limitation of iron uptake.

\section{ASSOCIATED CONTENT}

Supporting

Information

is available:

Methods and materials, purification and gel filtration data, additional structural images and tables, and representative partial sequence alignments

(PDF)

\section{AUTHOR INFORMATION}

\section{Corresponding Author}

*E-mail: smitha@umbc.edu. Phone: 410-455-1985

ORCID

Aaron T. Smith: 0000-0002-9332-8683

\section{Funding Sources}

No competing financial interests have been declared.

This work was supported by start-up funds from the University of Maryland, Baltimore County (A. T. S.), and in part by NIH-NIGMS T32 GM066706 (A. E. S.)

This research used resources of the Advanced Photon Source, a U.S. Department of Energy (DOE) Office of Science User Facility operated for the DOE Office of Science by Argonne National Laboratory under Contract No. DEAC02-06CH11357. Use of the LS-CAT Sector 21 was supported by the Michigan Economic Development Corporation and the Michigan Technology Tri-Corridor (Grant 085P1000817).

\section{ACKNOWLEDGMENT}

The authors wish to thank Zdzislaw Wawrzak for his knowledgeable help.

\section{ABBREVIATIONS}

ASU, asymmetric unit; Feo, ferrous iron transport system; GDP, guanosine-5'-diphosphate; GMP-PNP, 5'-guanylyl imidodiphosphate; GTP, guanosine-5'-triphosphate; MR, molecular replacement; NMR, nuclear magnetic resonance; SAD, single-wavelength anomalous dispersion; SH3, Srchomology 3-like fold

\section{REFERENCES}

1. Andrews, S. C.; Robinson, A. K.; RodríguezQuiñones, F., FEMS Microbiol Rev. 2003, 27, 215237.

2. Ganz, T., Cell Metab. 2008, 7, 288-290.

3. Podschun, R.; Ullmann, U., Clin. Microbiol. Rev. 1998, 11 (4), 589-603.

4. Li, B.; Zhao, Y.; Liu, C.; Chen, Z.; Zhou, D., Future Microbiol. 2014, 9 (9), 1071-1081.

5. Cartron, M. L.; Maddocks, S.; Gillingham, P.; Craven, C. J.; Andrews, S. C., Biometals 2006, 19, 143-157.

6. Sestok, A. E.; Linkous, R. O.; Smith, A. T., Metallomics 2018, 10, 887-898.

7. Smith, A. T.; Sestok, A. E., Protein Expr. Purif. 2018, 142, 1-7.

8. Nikaido, K.; Liu, P.-Q.; Ames, G. F.-L., J. Biol. Chem. 1997, 272 (44), 27745-27752.

9. $\quad$ Benabdelhak, H.; Schmitt, L.; Horn, C.; Jumel, K.; Blight, M. A.; Holland, I. B., Biochem. J. 2005, 386, 489-495.

10. Scherer, J.; Nies, D. H., Mol. Microbiol. 2009, 73 (4), 601-621.

11. Smith, A. T.; Ross, M. O.; Hoffman, B. M.; Rosenzweig, A. C., Biochemistry 2017, 56, 85-95.

12. Seyedmohammad, S.; Born, D.; Venter, H., Protein Expr. Purif. 2014, 101, 138-145. 
13. Lau, C. K. Y.; Ishida, H.; Liu, Z.; Vogel, H. J., J. Bacteriol. 2013, 195 (1), 46-55.

14. Lau, C. K. Y.; Krewulak, K. D.; Vogel, H. J., FEMS Microbiol. Rev. 2016, 40, 273-298.

15. Su, Y.-C.; Chin, K.-H.; Hung, H.-C.; Shen, G.-H.; Wang, A. H.-J.; Chou, S.-H., Acta. Cryst. F 2010, 66 (6), 636-642.

16. Hildebrand, J. D.; Taylor, J. M.; Parsons, J. T., Mol. Cell. Biol. 1996, 16 (6), 3169-3178.

17. Whisstock, J. C.; Lesk, A. M., Trends Biochem. Sci. 1999, 24 (1), 132-133.

18. Wylie, G. P.; Rangachari, V.; Bienkiewicz, E. A.; Marin, V.; Bhattacharya, N.; Love, J. F.; Murphy, J. R.; Logan, T. M., Biochemistry 2005, 44, 40-51.

19. Liu, C.; Mao, K.; Zhang, M.; Sun, Z.; Hong, W.; Li, C.; Peng, B.; Chang, Z., J. Biol. Chem. 2008, 283 (4), 2439-2453.

20. Saksela, K.; Permi, P., FEBS Lett. 2012, 586 (17), 2609-2614.

21. Vajda, S.; Yueh, C.; Beglov, D.; Bohnuud, T.; Mottarella, S. E.; Xia, B.; Hall, D. R.; Kozakov, D., Proteins: Struct., Funct., Bioinf. 2017, 85 (3), 435444.
22. Kozakov, D.; Hall, D. R.; Xia, B.; Porter, K. A.; Padhorny, D.; Yueh, C.; Beglov, D.; Vajda, S., Nat. Protoc. 2017, 12 (2), 255-278.

23. Hung, K. W.; Chang, Y. W.; Eng, E. T.; Chen, J. H.; Chen, Y. C.; Sun, Y. C.; Hsiao, C. D.; Dong, G.; Spasov, K. A.; Unger, V. M.; Huang, T. H., J. Struct. Biol. 2010, 170 (3), 501-512.

24. Kammler, M.; Schön, C.; Hantke, K., J. Bacteriol. 1993, 175 (19), 6212-6219.

25. Weaver, E. A.; Wyckoff, E. E.; Mey, A. R.; Morrison, R.; Payne, S. M., J. Bacteriol. 2013, 195 (21), 4826-4835.

26. Kim, H.; Lee, H.; Shin, D., Biochem. Biophys. Res. Com. 2012, 423, 733-738.

27. Álvarez-Fraga, L.; Vázquez-Ucha, J. C.; MartínezGuitián, M.; Vallejo, J. A.; Bou, G.; Beceiro, A.; Poza, M., Virulence 2018, 9 (1), 496-509.

28. Kurochkina, N.; Guha, U., Biophys. Rev. 2013, 5 (1), 29-39. 
bioRxiv preprint doi: https://doi.org/10.1101/514059; this version posted January 8,2019 . The copyright holder for this preprint (which was not certified by peer review) is the author/funder. All rights reserved. No reuse allowed without permission.

\section{SYNOPSIS TOC}

The crystal structure of K. pneumoniae FeoA reveals a site for protein-protein interactions mediated by a prokaryotic SH3like domain. Peptide docking implicates this location as the site of interaction between FeoA and FeoB. These proteinprotein interactions are likely utilized to control ferrous iron uptake, a key nutrient acquisition pathway used by pathogenic bacteria to establish infection in acidic and/or anaerobic niches within human hosts.

Image for Table of Contents Usage only:

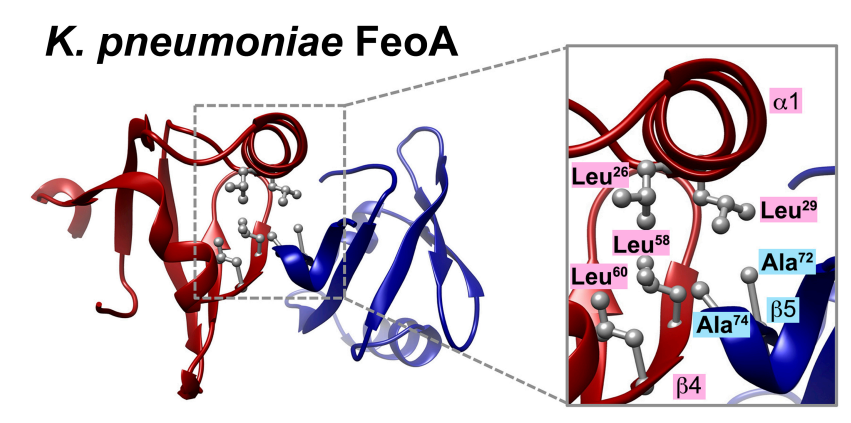

